\title{
Triptolide Inhibits the Biological Processes of HUVEC and HepG2 Cells via the Serine Palmitoyltransferase Long Chain Base Subunit 2/sphingosine-1-phosphate Signaling Pathway
}

qinyou tan

Guilin Medical University Affiliated Hospital https://orcid.org/0000-0001-6349-3453

shengnan zhu

Guilin Medical University

mingfei zhu

Guilin Medical University

lingyue huang

Guilin Medical University

siyuan $\mathrm{Xu}$

Guilin Medical University

yuqin luo

Guilin Medical University

juan xiao

Guilin Medical University Affiliated Hospital

huazhen su

Guilin Medical University Affiliated Hospital

shaoyuan huang

Guilin Medical University Affiliated Hospital

Iulu jia ( $\square$ jialuluedu@163.com )

Guilin Medical University Affiliated Hospital https://orcid.org/0000-0003-1616-896X

\section{Research Article}

Keywords: Triptolide, Serine palmitoyltransferase long chain base subunit 2, S1P receptors, Sphingosine1-phosphate

Posted Date: June 22nd, 2021

DOl: https://doi.org/10.21203/rs.3.rs-629932/v1 
License: (c) (i) This work is licensed under a Creative Commons Attribution 4.0 International License. Read Full License 
1

2

3

4

\title{
Triptolide inhibits the biological processes of HUVEC and
}

\section{HepG2 cells via the serine palmitoyltransferase long chain base}

\section{subunit 2/sphingosine-1-phosphate signaling pathway}

\author{
Qinyou Tan ${ }^{1,2,3}$, Shengnan Zhu ${ }^{1,2,3}$, Mingfei Zhu ${ }^{1,2,3}$, Lingyue Huang ${ }^{1,2,3}$, Siyuan $\mathrm{Xu}^{1,2,3}$, Yuqin \\ Luo $^{1,2,3}$, Juan Xiao ${ }^{2,3}$, Huazhen $\mathrm{Su}^{1}$, Shaoyuan Huang ${ }^{1}$, Lulu Jia ${ }^{1,2,3^{*}}$
}

\section{*Correspondence: jialuluedu@163.com}

${ }^{1}$ Clinical Pharmacy \& Pharmacology Research Institute, the Affiliated Hospital of Guilin Medical University, Guilin 541001, China

${ }^{2}$ Laboratory of Hepatobiliary and Pancreatic Surgery, the Affiliated Hospital of Guilin Medical University, Guilin 541001, China

${ }^{3}$ China-USA Lipids in Health and Disease Research Center, Guilin Medical University, Guilin 541001, China

\begin{abstract}
Background: Hepatocellular carcinoma is a cancer that has a high incidence in men, and its incidence is increasing year by year. Studies show that angiogenesis plays an important role in the formation of tumors, not only providing nutrients to tumor cells, but also closely related to tumor growth and metastasis. So, how to find new anti-vascular and anti-tumor targets for the pathogenesis of liver cancer is a key issue that needs to be resolved.

Methods: After treating Human umbilical vein endothelial cells (HUVEC) and HepG2 cells with different concentrations of triptolide (TP), the relationship between TP's anti-vascular and anti-tumor activities and sphingolipids was investigated respectively. Then, the three-dimensional co-culture model was used to explore the correlation between HUVEC and HepG2 cells, and to find the relationship between it and sphingolipids.

Results: This study explored the effects of TP on the proliferation, migration, adhesion and tube formation of HUVEC cells, as well as the effects on the proliferation, migration and invasion of HepG2 cells. And through the PCR Array assay to screen the changes of related sphingolipid genes, it was found that serine palmitoyltransferase long chain base subunit 2 (SPTLC2) was most likely to be related to the effect of TP. In further transfection experiments, it was found that down-regulation of SPTLC2 can inhibit the proliferation, migration, adhesion and tube formation of HUVEC cells, and down-regulation of SPTLC 2 can also inhibit the proliferation, migration and invasion of HepG2 cells. The up-regulation of SPTLC2 has opposite effects in these two cell lines. In the three-dimensional co-culture model of HUVEC and HepG2 cells, it was found for the first time that HUVEC cells can promote the biological process of HepG2 cells. It was found through enzyme linked immunosorbent assay (ELISA) and western blot experiments that it may be achieved through the sphingosine-1-phosphate (S1P)/S1P receptors (S1PRs) pathway. Finally, we found that the promotion effect of HUVEC cells on HepG2 cells was significantly inhibited after HUVEC cells were treated with TP.

Conclusions: These data confirmed that the level of SPTLC2 may be related to the anti-vascular
\end{abstract}


and anti-tumor effects of TP. The data also showed that there was a correlation between the viability of HepG2 cells and HUVEC cells, which may be related to the expression of S1P/S1PR . Ultimately, these data may help discover new anti-tumor targets.

Keywords: Triptolide, Serine palmitoyltransferase long chain base subunit 2, S1P receptors, Sphingosine-1-phosphate

\section{Introduction}

Hepatocellular carcinoma is a cancer that has a high incidence in men, and its incidence is increasing year by year [1]. The pathogenesis of liver cancer is a very complicated process composed of a series of related links mediated by various risk factors such as chronic viral hepatitis, alcohol abuse, non-alcoholic steatohepatitis and type 2 diabetes [2]. Studies show that angiogenesis plays an important role in the formation of tumors, not only providing nutrients to tumor cells, but also closely related to tumor growth and metastasis [3]. In addition, these new vascular tissues can provide blood and nutrients for the continued growth of tumors, and tumor tissues can further promote the regeneration of blood vessels through a variety of ways, thereby forming a vicious circle, so modern medicine believes that cutting off the vascular tissues of tumors can "starved to death" tumor. So, how to find new anti-vascular and anti-tumor targets for the pathogenesis of liver cancer is a key issue that needs to be resolved. Only by cutting off the nutritional supply of tumor tissue and inhibiting the further growth of tumors can liver cancer be fundamentally overcome.

In recent years, with the further study, it is found that in addition to being the basic component of cell membrane, sphingolipids also participate in a variety of signal transduction pathways and play an important role in the occurrence and development of various diseases. The metabolism of sphingolipids is a key pathway in cancer biology, and its metabolites ceramide, sphingosine and S1P together regulate tumor cell death, proliferation and drug resistance, as well as angiogenesis and inflammation [4]. Ceramide is produced by the hydrolysis of sphingolipids, which can also de novo synthesis of ceramide by the precursor dihydroceramide, which is converted to ceramide by dihydroceramide desaturase to induce tumor cell apoptosis [5]. Ceramide is hydrolyzed by a ceramidase to produce sphingosine, which is phosphorylated by sphingosine kinases (SK1 and SK2) to produce S1P. S1P is dephosphorylated by S1P phosphatase 1 and 2, and is degraded by S1P lyase, which cleaves S1P to produce phosphoethanolamine and hexadecenal. In addition to intracellular targets, S1P also binds to and activates the G protein-coupled receptor family S1P receptor 1-5 (S1PR1-5), which regulates the biological activity of cells. Studies in many cancer cell lines indicate that S1P induces proliferation and inhibits ceramide-induced apoptosis. Ceramide is a key factor in sphingolipid metabolism, and serine palmitoyltransferase (SPT) is a key enzyme for de novo synthesis of ceramide. SPT in mammals is a heterodimer composed of two subunits, namely SPTLC1 and SPTLC2, and SPTLC3 is the third subunit found in 2009 [6]. SPT is a class of enzyme with pre-inflammatory and pre-apoptotic properties that can be involved in subsequent responses by activating multiple protein kinases and phosphatases downstream of the inflammatory response, or by generating S1P as a second messenger [7].

$\mathrm{TP}$ is one of the main active ingredients extracted from the roots, stems and leaves of tripterygium wilfordii. It is a small molecule compound with anti-tumor, anti-angiogenesis, anti-inflammatory and pro-apoptotic effects [8]. TP has anti-cancer effect against liver [9,10], ovarian [11-14], lung [15-17], gastric [18,19], breast [20,21]. 
Based on the above facts, this experiment explores the effects of TP on liver cancer from the three perspectives of anti-vascularity, tumor suppression and tumor microenvironment, and finds the connection between TP's anti-liver cancer effect and sphingolipid. In this study, we first studied the effect of TP on HUVEC cells and its possible mechanism, explored the effect of TP on angiogenesis, and looked for the effect of TP on angiogenesis new target. Secondly, we studied the effect of TP on hepatocellular carcinoma cell line HepG2, and further explored the new target of TP against liver cancer. As we all know, as one of the members of the tumor microenvironment, vascular endothelial cells not only form vascular nutrition tumor tissue, but also penetrate the entire tumor tissue. So does endothelial cell itself have a certain effect on tumor cells? Then, we further explored the interaction between HUVEC and HepG2 cells in the three-dimensional co-cultivation mode and its possible mechanism, and studied the influence of TP on the co-cultivation system.

\section{Materials and methods}

\section{Cell culture}

HUVEC cells were purchased from the North Branch of the Institute of Biotechnology, and HepG2 cells were purchased from the Chinese Academy of Sciences. The cells were cultured in DMEM supplemented with $10 \%$ FBS, $1 \%(\mathrm{v} / \mathrm{v})$ penicillin-streptomycin, and were maintained at $37^{\circ} \mathrm{C}$ in a humidified $5 \% \mathrm{CO}_{2}$ incubator (Thermo Electron, 3111 , USA).

\section{Cell proliferation assay}

Cell viability was determined by the CCK-8 assay. In monoculture systems, HUVEC and HepG2 cells were adjusted to a density of $4 \times 10^{4}$ cells $/ \mathrm{ml}$, plated into 96 -well plates $(100 \mu \mathrm{l} / \mathrm{well})$. The HUVEC cells were treated with TP $(0,12.5,25,50 \mathrm{nM})$, DMSO (negative control) and endostatin (positive control, $8 \mathrm{mg} / \mathrm{L}$ ) for 24,48 , and $72 \mathrm{~h}$. The HepG2 cells were treated with TP $(0,1,2,4$ $\mu \mathrm{M})$ and DMSO (negative control) for 24 and $48 \mathrm{~h}$. In monoculture transfection system, HUVEC and HepG2 cells were divided into small interfering RNA of SPTLC2 group (siR-SPTLC2), SPTLC2 plasmid group (SPTLC2), and its negative control group (siR-NC/SPTLC2-NC) and blank control group (Blank). In addition, HUVEC and HepG2 cells were treated with $25 \mathrm{nM}$ and 2 $\mu \mathrm{M}$ TP for $24 \mathrm{~h}$. In co-culture systems, $2 \times 10^{3} \mathrm{HepG} 2$ cells $(200 \mu \mathrm{l} /$ well) were added to the upper chamber of the 24-well co-culture chamber, and $5 \times 10^{3}$ HUVEC cells ( $500 \mu 1 /$ well) were added to the lower chamber (Co-culture), put DMEM $(500 \mu \mathrm{l} /$ well) containing $10 \%$ FBS in the lower chamber as a control group (Non-co-culture), cells were incubated for 1 to 4 days. In co-culture dosing system, the HUVEC cells were treated with TP $(0,12.5,25,50 \mathrm{nM})$ and DMSO (negative control) for 24h, and then the HUVEC cells were rinsed with PBS. These treated HUVEC cells were cultured with HepG2 cells as described in co-culture systems for $24 \mathrm{~h}$.

In monoculture or monoculture transfection systems, the supernatant was removed and $100 \mu \mathrm{l}$ of CCK-8 medium (CCK-8 reagent : DMEM = $1: 10$ ) were added to the wells. In co-culture and co-culture dosing systems, transferred the upper chamber to a new 24-well plate containing $500 \mu \mathrm{l}$ of CCK-8 medium (CCK-8 reagent : DMEM = 1:10). After 1 to 4 hours, measure the absorbance at $450 \mathrm{~nm}$ with a microplate reader (Thermo, Multiskn Spectrum 1500, USA).

\section{Cell migration and invasion assay}

The migration ability of the HUVEC and HepG2 cells was determined using a 24-well 
two-compartment transwell assay. Cells in monoculture, monoculture transfection, co-culture and co-culture dosing systems were cultured as described in the proliferation assay. The each group of HUVEC and HepG2 cells $\left(3 \times 10^{4} / 200 \mu \mathrm{l}\right)$ were resuspended in serum-free DMEM. $200 \mu \mathrm{l}$ of the cell suspension was added to the upper chamber of the transwell, and $500 \mu$ of the complete medium was added to the lower chamber. After $24 \mathrm{~h}$, the upper chamber was taken out and washed with PBS three times, $4 \%$ tissue cell fixative was fixed for $1 \mathrm{~h}$, washed again with PBS for 3 times, $0.1 \%$ crystal violet stained for $30 \mathrm{~min}$, washed again with PBS for 3 times, gently wipe the cells inside the chamber with a cotton swab, and finally photographed with a microscope ( $\times 100)$. After the end of the photographing, each group of chambers was decolorized for $5 \mathrm{~min}$ in $500 \mu \mathrm{l}$ of a $10 \%(\mathrm{v} / \mathrm{v})$ acetic acid solution, and the absorbance was measured at $550 \mathrm{~nm}$.

The cell invasion assay was similar to the cell migration assay except that the transwell membrane was pretreated with matrigel and the HepG2 cells density was adjusted to $3 \times 10^{5} / 200$ $\mu \mathrm{l}$.

\section{Cell adhesion assay}

The pre-cooled matrigel ( $50 \mu \mathrm{l} /$ well) was placed in a pre-cooled 96 -well plate. $2 \%$ BSA $(100 \mu \mathrm{l})$ was added to each well and blocked for $1 \mathrm{~h}$. HUVEC cells in monoculture and monoculture transfection systems were cultured as described in the proliferation assay. HUVEC cells $\left(3 \times 10^{4} / 100 \mu \mathrm{l}\right)$ were seeded in 96 -well plate. After $1 \mathrm{~h}$, the supernatant was removed and $100 \mu \mathrm{l}$ of CCK-8 medium (CCK-8 reagent : DMEM $=1: 10$ ) were added to the wells. After 1 to $4 \mathrm{~h}$, measure the absorbance at $450 \mathrm{~nm}$ with a microplate reader (Thermo, Multiskn Spectrum 1500, USA).

\section{Cell tube formation assay}

Matrigel was plated in 96-well culture plates and allowed to polymerise at $37{ }^{\circ} \mathrm{C}$ in $5 \% \mathrm{CO} 2$ humidified for $30 \mathrm{~min}$. HUVEC cells were digested with $0.25 \%$ trypsin to prepare a cell suspension, adjusted to a cell density of $8 \times 10^{4} / 100 \mu \mathrm{l}$, and added to a 96 -well plate plated with matrigel, $100 \mu \mathrm{l}$ of cell suspension per well, take pictures after $4-8 \mathrm{~h}(\times 100)$. The quantification of tube formation is carried out by counting the number of branch points.

\section{PCR-array and RT-PCR assay}

Total RNA from cells was prepared using TRNzol Universal (TIANGEN, Beijing, China) according to the manufacturer's protocol. Absorbance was measured at 260 and $280 \mathrm{~nm}$ to assess the quantity and purity of RNA. The cDNA was prepared from total RNA $(1 \mu \mathrm{g})$ with a reverse transcriptase (RT) Primer Mix using the PrimeScript RT reagent Kit with gDNA Eraser (TIANGEN, Beijing, China) according to the manufacturer's instructions. Genetic screening was performed using RT $^{2}$ Profiler PCR Arrays kit (QIAGEN, Maryiang, USA). The PCR Array plate contains 48 genes, including 40 target genes and 8 control genes. Subsequent PCR amplification was carried out using a Bio-Rad CFX Manager 3.1 system (Bio-Rad, Hercules, CA) under the following conditions: 40 cycles at $95{ }^{\circ} \mathrm{C}$ for $15 \mathrm{~s}$ and at $60{ }^{\circ} \mathrm{C}$ for $60 \mathrm{~s}$. Amplified products were monitored by measuring the increase of the dye intensity of the SYBR Green that binds to double-strand DNA amplified by PCR. GAPDH was used as an internal control. GAPDH forward primer (5'-3'): CAGGAGGCATTGCTGATGAT; GAPDH reversed primer (5'-3'): GAAGGCTGGGGCTCATTT; SPTLC2 forward primer (5'-3'): CAGATTGCTTGAGGCCAGGA 
AGTTC; SPTLC2 reversed primer (5'-3'): AGTGGTGTGATCTTGGCTCATTGC.

\section{Western blotting}

After the HUVEC and HepG2 cells were treated with the drug or transfected under the above conditions, the protein was extracted for subsequent experiments. The cells were subsequently lysed with RIPA buffer (Solarbio, China), according to the manufacturer's recommendations. Equivalent amounts of protein were separated by $10 \%$ SDS-PAGE and transferred to a PVDF membrane. The membrane blocked in 5\% nonfat milk in TBST for $1 \mathrm{~h}$ at room temperature and then incubated with primary antibodies at $4{ }^{\circ} \mathrm{C}$ overnight. The primary antibodies we use are anti-serine palmitoyltransferase antibody (abcam, ab23696), anti-S1P1 antibody (abcam, ab233386), anti-S1PR2 antibody (abcam, ab220173), and anti-S1P3 antibody (abcam, ab126622). The immunoblots were then incubated with a secondary antibody at room temperature. The antigen-antibody complex on the membrane was visualized using ECL plus and X-ray film. Finally, the protein concentration was analyzed using ImageJ.

\section{Enzyme linked immunosorbent assay}

In monoculture transfection system, HUVEC and HepG2 cells were cultured as described in the proliferation assay. After the cells were cultured for $24 \mathrm{~h}$, the supernatants were collected. In the co-culture system, $2 \times 10^{5} \mathrm{HepG} 2$ cells $(2 \mathrm{ml} /$ well) were added to the upper chamber of the 6-well co-culture chamber, and $1 \times 10^{5}$ HUVEC cells $(1 \mathrm{ml} /$ well) were added to the lower chamber. After the cells were co-cultured for 1 to 4 days, the supernatants were collected. It was detected by sphingosine-1-phosphate ELISA kit (Echelon, Salt Lake, USA) according to the manufacturer's protocol.

\section{Statistical analysis}

All data were represented by mean \pm standard deviation (SD). GraphPad Prism 5.0 software was applied to statistical analysis, and significance between groups was ascertained by one-way ANOVA compared with least significant difference. When the p-value was less 0.05 , the analysis was accepted as statistical difference.

\section{Results}

\section{TP inhibited HUVEC cells proliferation, migration, adhesion and angiogenesis}

Firstly, we assessed the effects of TP on HUVEC cells proliferation. The data (Figure 1A) showed that TP inhibited the proliferation of HUVEC cells in a dose and time dependent manner, endostatin (positive control) could significantly inhibit the proliferation of HUVEC cells, DMSO (negative control) had no significant effect on the proliferation of HUVEC cells. After treating HUVEC cells with TP $(0,12.5,25,50 \mathrm{nM})$, DMSO and endostatin $(8 \mathrm{mg} / \mathrm{L})$ for $24 \mathrm{~h}$, the migration (Figure 1B), adhesion (Figure 1C) and angiogenesis (Figure 1D) of HUVEC were measured respectively. The migration capacity of HUVEC cells was measured in a 24-well transwell chamber, and the migrated cells in each chamber were decolorized (500 $\mu 1,10 \%$ acetic acid) and their absorbance was measured at $550 \mathrm{~nm}$ to reflect the number of migrated cells. The data showed that TP inhibited HUVEC cells migration, adhesion and angiogenesis in a dose dependent manner, endostatin (positive control) could significantly inhibit HUVEC cells migration, adhesion and angiogenesis, DMSO (negative control) had no significant effect on 
HUVEC cells migration, adhesion and angiogenesis.

\section{TP down-regulated the expression of SPTLC2 in HUVEC cells}

The data (Figure $2 \mathrm{~A})$ showed that TP $(25 \mathrm{nM})$ had a regulatory effect on a variety of nerve spingolipid genes, but among all the genes SPTLC2 had the largest variation range which was likely to be a new target of TP, the data results were the absolute value of $\Delta \mathrm{Ct}$. Therefore, we chose SPTLC2 for subsequent studies. HUVEC cells were treated with TP $(25 \mathrm{nM})$ for $24 \mathrm{~h}$, and the expression of SPTLC2 mRNA in HUVEC cells was detected by RT-PCR assay, and the data (Figure 2B) showed that TP could significantly inhibit the expression of SPTLC2 in HUVEC cells. HUVEC cells were treated with TP $(0,12.5,25,50 \mathrm{nM})$ and DMSO (negative control) for $24 \mathrm{~h}$, and the expression of SPTLC2 protein was detected by western blot assay. The data (Figure 2C) showed that TP could inhibit the expression of SPTLC2 in HUVEC cells in a dose-dependent manner, DMSO had no significant effect on SPTLC2.

SPTLC2 affected the proliferation, migration, adhesion, angiogenesis and S1P production of HUVEC cells

The data (Figure 3A) showed that the expression of SPTLC2 protein was significantly inhibited in the siR-SPTLC2 (small interfering RNA of SPTLC2) group, the expression of SPTLC2 protein was significantly increased in the SPTLC2 (SPTLC2 plasmid) group, the expression of SPTLC2 protein was not significantly changed in siR-NC (small interfering RNA negative control of SPTLC2) and SPTLC2-NC (plasmid negative control of SPTLC2) groups. After HUVEC cells were transfected with siR-NC, siR-SPTLC2, SPTLC2-NC and SPTLC2, their proliferation (Figure 3B), migration (Figure 3C), adhesion (Figure 3D), angiogenesis (Figure 3E) and S1P production (Figure 3F) were measured respectively. The results showed that the siR-SPTLC2 could significantly inhibit the proliferation, migration, adhesion, angiogenesis and S1P production of HUVEC cells, the SPTLC 2 could significantly increase the proliferation, migration, adhesion, angiogenesis and S1P production of HUVEC cells, siR-NC and SPTLC2-NC had no significant effect on the proliferation, migration, adhesion, angiogenesis and S1P production of HUVEC cells. On this basis, the proliferation, migration, adhesion and angiogenesis of HUVEC cells in each group was further inhibited after treated with TP $(25 \mathrm{nM})$. Based on this, we believe that SPTLC2 may regulate various biological processes of HUVEC cells by regulating the production of S1P.

TP inhibited proliferation, migration, invasion, SPTLC2 mRNA and protein expression in HepG2 cells

Figure 4A presented that TP inhibited the proliferation of HepG2 cells in a dose and time dependent manner, DMSO had no significant effect on the proliferation of HepG2 cells. After treating HepG2 cells with TP $(0,1,2,4 \mu \mathrm{M})$ and DMSO (negative control) for $24 \mathrm{~h}$, the migration (Figure 4B), invasion (Figure 4C), SPTLC2 mRNA (Figure 4D) and protein (Figure 4E) expression of HepG2 were measured respectively. The migration or invasion capacity of HepG2 cells was measured in a 24-well transwell chamber, and the migrated or invaded cells in each chamber were decolorized ( $500 \mu 1,10 \%$ acetic acid) and their absorbance was measured at $550 \mathrm{~nm}$ to reflect the number of migrated or invaded cells. The data showed that TP inhibited HepG2 cells migration, invasion, SPTLC2 mRNA and protein expression in a dose dependent manner, DMSO had no significant effect on HepG2 cells migration, invasion, SPTLC2 mRNA and protein 
expression.

SPTLC2 affected the proliferation, migration, invasion and S1P production of HepG2 cells The data (Figure 5A) showed that the expression of SPTLC2 protein was significantly inhibited in the siR-SPTLC2 (small interfering RNA of SPTLC2) group, the expression of SPTLC2 protein was significantly increased in the SPTLC2 (SPTLC2 plasmid) group, the expression of SPTLC2 protein was not significantly changed in siR-NC (small interfering RNA negative control of SPTLC2) and SPTLC2-NC (plasmid negative control of SPTLC2) groups. After HepG2 cells were transfected with siR-NC, siR-SPTLC2, SPTLC2-NC and SPTLC2, their proliferation (Figure 5B), migration (Figure 5C), invasion (Figure 5D) and S1P production (Figure 5E) were measured respectively. The results showed that the siR-SPTLC2 could significantly inhibit the proliferation, migration, invasion and S1P production of HepG2 cells, the SPTLC2 could significantly increase the proliferation, migration, invasion and S1P production of HepG2 cells, siR-NC and SPTLC2-NC had no significant effect on the proliferation, migration, invasion and S1P production of HepG2 cells. On this basis, the proliferation, migration, invasion of HepG2 cells was further inhibited after treated with TP $(2 \mu \mathrm{M})$. Based on this, we believe that SPTLC2 may regulate various biological processes of HepG2 cells by regulating the production of S1P.

HUVEC cells may induce proliferation, migration and invasion of HepG 2 cells via the S1P-S1PR $_{S}$ pathway

In order to verify the effect of HUVEC cells on proliferation, migration and invasion of HepG2 cells, HUVEC cells and HepG2 cells were co-cultured to further detect the proliferation, migration and invasion of HepG2 cells. In the non-co-culture or control group, HepG2 cells were added to the upper chamber of the 24-well co-culture chamber, and DMEM was added to the lower chamber. In the co-culture group, HepG2 cells were added to the upper chamber of the 24-well co-culture chamber, and HUVEC cells were added to the lower chamber. The co-culture time of HUVEC and HepG2 cells were set to 1 to 4 days, the data (Figure 6A) showed that HUVEC cells can promote the proliferation of $\mathrm{HepG} 2$ cells, and their proliferative effects increase as co-culture time increases. HUVEC and HepG2 were co-cultured for $24 \mathrm{~h}$, the data showed that HUVEC cells can promote the migration (Figure 6B) and invasion (Figure 6C) of HepG 2 cells $(\times 100)$. HUVEC and HepG2 were co-cultured for 1 to 4 days, the data showed that the content of S1P (Figure 6D) in the co-culture system and the expression of S1PR1 and S1PR2 protein (Figure 6E) in HepG2 cells increased, while the expression of S1PR3 protein (Figure 6E) decreased gradually.

TP inhibited the proliferation, migration and invasion of HepG2 cells induced by HUVEC cells

The HUVEC cells were treated with TP $(0,12.5,25,50 \mathrm{nM})$ for $24 \mathrm{~h}$, the medium was changed to remove the drug influence, and the treated HUVEC cells were co-cultured with HepG2 cells through transwell co-culture chamber. HepG2 cells were added in the upper compartment, and treated HUVEC cells were added in the lower compartment. The data showed that when HUVEC cells were treated with TP, its promoting effects on proliferation (Figure 7A), migration (Figure 7B) and invasion (Figure 7C) of HepG2 cells were significantly inhibited. 


\section{Discussion}

Angiogenesis is known to play an important role in tumor growth and metastasis. The newly formed vascular tissue can not only metastasize tumor cells, but also provide continuous nutritional supply to tumor tissue [22]. Therefore, modern medicine believes that the removal of tumor blood vessel tissue can "starve" the tumor. Therefore, in addition to inhibiting the occurrence and development of tumor tissue, the treatment of tumor is more important to control the birth of blood vessels. TP is one of the most popular anti-tumor drugs in recent years. It not only inhibits tumor angiogenesis, but also inhibits various tumor biological processes[24-26]. In recent years, with the deepening of research, it has been found that nerve sphingolipids are not only the basic components of cell membranes, but also participate in a variety of signal transduction pathways, and play an important role in the occurrence and development of various diseases, especially tumors [4]. Based on these facts, we firstly studied the mechanism of anti-vascular and anti-liver cancer of TP. Our experimental data showed that TP could down-regulate the expression of SPTLC2 in HUVEC and HepG2 cells, and further cell transfection experiments showed that the biological behavior of the two cell lines was significantly inhibited after SPTLC2 knockdown, while the biological behavior of the two cell lines was significantly promoted after SPTLC2 up-regulation. In addition, down-regulation of SPTLC2 inhibited the production of S1P in both cell lines, while up-regulation of SPTLC2 increased the production of S1P. Based on the above experimental results, we believe that SPTLC2 is likely to be a new anti-vascular and anti-tumor target of TP, and the regulatory effect of SPTLC2 on cells is likely to be realized by indirectly regulating the production of S1P. We believe that SPTLC2 is likely to be a new target for tumor inhibition from nerve spingolipin-related pathways. However, this anti-tumor mechanism of TP has only been verified in HepG2 cells, and its universality in other tumors has yet to be verified by a large number of subsequent experiments.

Angiogenesis is associated with many tumors, especially solid tumors, such as liver and breast cancer. As a member of the tumor microenvironment, vascular endothelial cells can not only form vascular nutrient tumor tissue, but also penetrate the whole tumor tissue. Therefore, does the vascular endothelial cells themselves have a certain promotion effect on tumor cells. Then, we designed co-culture experiments of HUVEC and HepG2 cells. Our datas showed that the proliferation, migration and invasion of HepG2 cells co-cultured with HUVEC cells were significantly enhanced compared with HepG2 cells cultured alone. Mechanism studies have shown that HUVEC cells may secrete S1P and then act on S1PR on HepG2 cells. This discovery further opens up the research field of the effect of vascular endothelial cells on tumor cells, and enables us to find that vascular endothelial cells can also promote the biological process of tumor cells, which provides a theoretical basis for further research on the effect between vascular endothelial cells and tumor cells. However, this conclusion has only been verified in HepG2 cells, and its effect in other tumor cells and animal experiments needs to be further studied.

In conclusion, the results of this study indicated that TP inhibited the biological process of HUVEC and HepG2 cells by regulating the SPTLC2-S1P axis, and found that HUVEC cells could promote the biological behavior of HepG2 cells. This finding is helpful to further understand the anti-vascular and anti-tumor effects of TP from the nerve sphingolipids pathway, and to further discover the role of nerve sphingolipids in the occurrence and development of tumors. 


\title{
Study strengths and limitations
}

This study explored the effects of TP on liver cancer from the perspectives of anti-vascular, anti-cancer and tumor microenvironment, and discovered the connection between the anti-liver cancer effect of TP and sphingolipids. New targets have been discovered for anti-vascular and anti-tumor research, which will further promote the study of tumorigenesis mechanisms. This study has several limitations. The regulatory effect of SPTLC2 in other tumors needs further experimental verification, and the relationship between TP's anti-tumor effect and SPTLC2 in vivo also needs further experimental research.

\section{Conclusions}

These data confirm that the level of SPTLC2 may be related to the anti-vascular and anti-tumor effects of TP. The data also showed that there is a correlation between the viability of HepG2 cells and HUVEC cells, which may be related to the expression of S1P/S1PR. Ultimately, these data may help discover new anti-tumor targets.

\begin{abstract}
Abbreviations
HUVEC: Human umbilical vein endothelial cells; TP: Triptolide; PCR: polymerase chain reaction; S1P: sphingosine-1-phosphate; S1PRs: sphingosine-1-phosphate receptors; SPTLC2: Serine palmitoyltransferase 2; siRNA: small interfering RNA; OD: optical density; ELISA: enzyme-linked immuno sorbent assay
\end{abstract}

\section{Acknowledgements}

Thanks to the scientific research team of the Hepatobiliary and Pancreatic Surgery Laboratory of Guilin Medical College Affiliated Hospital. Special thanks to Dr. Xiao Juan for his guidance on this paper.

\section{Authors' contributions}

LL-J and QY-T conceived and designed the experiments; LL-J and MF-Z performed the experiments; LL-J and QY-T analyzed the data; LL-J and QY-T wrote the paper. The author(s) read and approved the final manuscript.

\section{Funding}

This study was supported by the National Natural Science Foundation of China (Grants Nos. 81360665, 81760747 ) and also partially supported by the Guangxi Municipal Natural Science Foundation Grants 2015GXNSFAA139114 and 2018GX NSFAA050147. The present study was also supported in part by the Lijiang Scholar Award in Guilin, Hundred Talents Program "the Introduction of Overseas High-Level Talents in Colleges and Universities in Guangxi”, and the High Level of Innovation Team and Outstanding Scholars Program in Colleges and Universities in Guangxi.

\section{Availability of data and materials}

The data in this article is dependable. The original data is available from the corresponding authors.

\section{Ethics approval and consent to participate}

This manuscript does not contain any studies with human participants or animals performed by any of the authors.

\section{Consent for publication}

Authors provide formal consent to publish work. 


\section{Competing interests}

The authors declare no conflict of interest

\section{References}

1. Hsieh YP, Wu KJ, Chen HM, Deng YT. Arecoline activates latent transforming growth factor $\beta 1$ via mitochondrial reactive oxygen species in buccal fibroblasts: Suppression by epigallocatechin-3-gallate. J Formos Med Assoc. 2018, 117(6): 527-534.

2. Fedeles BI, Chawanthayatham S, Croy RG, Wogan GN, Essigmann JM. Early detection of the aflatoxin B(1) mutational fingerprint: A diagnostic tool for liver cancer. Mol Cell Oncol. 2017, 4(4): e1329693.

3. Dong G, Lin XH, Liu HH, Gao DM, Cui JF, Ren ZG, Chen RX. Intermittent hypoxia alleviates increased VEGF and pro-angiogenic potential in liver cancer cells.Oncol Lett. 2019, 18(2): 1831-1839.

4. Lewis CS, Voelkel-Johnson C, Smith CD. Targeting Sphingosine Kinases for the Treatment of Cancer. Adv Cancer Res. 2018, 140: 295-325.

5. Kolesnick R, Fuks Z. Radiation and ceramide-induced apoptosis. Oncogene. 2003, 22(37): 5897-906.

6. Mandon EC, Ehses I, Rother J, van Echten G, Sandhoff K. Subcellular localization and membrane topology of serine palmitoyltransferase, 3-dehydrosphinganine reductase, and sphinganine N-acyltransferase in mouse liver. J Biol Chem. 1992, 267(16): 11144-8.

7. Ohta E, Ohira T, Matsue K, Ikeda Y, Fujii K, Ohwaki K, Osuka S, Hirabayashi Y, Sasaki M. Analysis of development of lesions in mice with serine palmitoyltransferase (SPT) deficiency -Sptlc2 conditional knockout mice-. ExpAnim. 2009, 58(5): 515-24.

8. Hu H, Huang G, Wang H, Li X, Wang X, Feng Y, Tan B, Chen T. Inhibition effect of triptolide on human epithelial ovarian cancer via adjusting cellular immunity and angiogenesis. Oncol Rep. 2018, 39(3): 1191-1196.

9. Sun YY, Xiao L, Wang D, Ji YC, Yang YP, Ma R, Chen XH. Triptolide inhibits viability and induces apoptosis in liver cancer cells through activation of the tumor suppressor gene p53. Int J Oncol. 2017, 50(3): 847-852.

10. Li Y, Hu S. Triptolide sensitizes liver cancer cell lines to chemotherapy in vitro and in vivo. Panminerva Med. 2014, 56(3): 211-20.

11. Liu H, Shen M, Zhao, Ru D, Duan Y, Ding C, Li H. The Effect of Triptolide-Loaded Exosomes on the Proliferation and Apoptosis of Human Ovarian Cancer SKOV3 Cells. Biomed Res Int. 2019, 2019: 2595801

12. Huang G, Hu H, Zhang Y, Zhu Y, Liu J, Tan B, Chen T. Triptolide sensitizes cisplatin-resistant human epithelial ovarian cancer by inhibiting the phosphorylation of AKT. J Cancer. 2019, 10(13): 3012-3020.

13. Wang R, Ma X, Su S, Liu Y. Triptolide antagonized the cisplatin resistance in human ovarian cancer cell line A2780/CP70 via hsa-mir-6751. Future Med Chem. 2018, 10(16) :1947-1955.

14. Wang Y, Liu T, Li H. Enhancement of triptolide-loaded micelles on tumorigenicity inhibition of human ovarian cancer. J Biomater Sci Polym Ed. 2016, 27(7): 545-56.

15. Tong X, Jiang P, Li Y, Guo L, Zhang HM, Zhang BK, Yan M. Combined Treatment with Triptolide and Tyrosine Kinase Inhibitors Synergistically Enhances Apoptosis in Non-small Cell Lung Cancer H1975 Cells but Not H1299 Cells through EGFR/Akt Pathway. Chem Pharm Bull (Tokyo). 2019, 67(8): 864-871. 
16. Huang Y, Chen Z, Wang Y, Ba X, Huang Y, Shen P, Wang H, Tu S. Triptolide exerts an anti-tumor effect on non-small cell lung cancer cells by inhibiting activation of the IL-6/STAT3 axis. Int J Mol Med. 2019, 44(1): 291-300.

17. Mao X, Tong J, Wang Y, Zhu Z, Yin Y, Wang Y. Triptolide exhibits antitumor effects by reversing hypermethylation of WIF-1 in lung cancer cells. Mol Med Rep. 2018, 18(3):

3041-3049.

18. Xie M, Wu J, Ji L, Jiang X, Zhang J, Ge M, Cai X. Development of Triptolide Self-Microemulsifying Drug Delivery System and Its Anti-tumor Effect on Gastric Cancer Xenografts. Front Oncol. 2019, 9:978.

19. Teng F, Xu ZY, Lyu H, Wang YP, Wang LJ, Huang T, Sun JC, Zhu HT, Ni YX, Cheng $\mathrm{XD}$. [Triptolide reverses apatinib resistance in gastric cancer cell line MKN45 via inhibition of heat shock protein 70]. Zhonghua Zhong Liu Za Zhi. 2018, 40(2): 92-98.

20. Zheng W, Wang C, Ding R, Huang Y, Li Y, Lu Y. Triptolide-loaded nanoparticles targeting breast cancer in vivo with reduced toxicity. Int J Pharm. 2019, 572:118721.

21. Wu H, Sun T, Bi R. Inhibition of insulin-like growth factor 1 signaling synergistically enhances the tumor suppressive role of triptolide in triple-negative breast cancer cells. Oncol Lett. 2019, 18(1): 822-829.

22. Richert MM, Vaidya KS, Mills CN, et al. Inhibition of CXCR4 by CTCE-9908 inhibits breast cancer metastasis to lung and bone [J]. Oncol Rep. 2009, 21(3): 761-7.

23. Nagahashi M, Yamada A, Miyazaki H, et al. Interstitial Fluid Sphingosine-1-Phosphate in Murine Mammary Gland and Cancer and Human Breast Tissue and Cancer Determined by Novel Methods [J]. J Mammary Gland Biol Neoplasia. 2016, 21(1-2): 9-17.

24. Wang X, Zhang L, Duan W, et al. Anti-inflammatory effects of triptolide by inhibiting the NF-kB signalling pathway in LPS-induced acute lung injury in a murine model [J]. Mol Med Rep. 2014, 10(1): 447-52.

25. Chen X, Murakami T, Oppenheim JJ, Howard OM. Triptolide, a constituent of immunosuppressive Chinese herbal medicine, is a potent suppressor of dendritic-cell maturation and trafficking. Blood. 2005, 106(7): 2409-16

26. Yang S, Chen J, Guo Z, et al. Triptolide inhibits the growth and metastasis of solid tumors. Mol Cancer Ther. 2003, 2(1): 65-72. 35 . 


\section{Figure legends:}

Fig. 1 TP inhibited the proliferation, migration, adhesion and angiogenesis of HUVEC cells. (A) CCK-8 assay was used to determine HUVEC cells proliferation after treated with TP $(0,12.5,25$, $50 \mathrm{nM})$, DMSO and endostatin $(8 \mathrm{mg} / \mathrm{L})$ for 24,48 and $72 \mathrm{~h}$. After treating HUVEC cells with TP $(0,12.5,25,50 \mathrm{nM})$, DMSO and endostatin $(8 \mathrm{mg} / \mathrm{L})$ for $24 \mathrm{~h}$, the migration $(\mathrm{B})$, adhesion $(\mathrm{C})$ and angiogenesis (D) of HUVEC were measured respectively. ${ }^{*} \mathrm{P}<0.05$, ** $\mathrm{P}<0.01$ versus control group.

Fig. 2 TP significantly down-regulated the expression of SPTLC2 in HUVEC cells. (A) HUVEC cells were treated with TP $(25 \mathrm{nM})$ for $24 \mathrm{~h}$, and the changes of related genes were screened by PCR Array assay, the data results were the absolute value of $\triangle \mathrm{Ct}$. (B) HUVEC cells were treated with TP $(25 \mathrm{nM})$ for $24 \mathrm{~h}$, and the expression of SPTLC2 mRNA was detected by RT-PCR assay. (C) HUVEC cells were treated with TP $(0,12.5,25,50 \mathrm{nM})$ and DMSO (negative control) for 24 $\mathrm{h}$, and the expression of SPTLC2 protein in HUVEC cells was detected by western blot assay. * $\mathrm{P}<0.05$, ** $\mathrm{P}<0.01$ versus control group.

Fig. 3 SPTLC2 can affect the proliferation, migration, adhesion, angiogenesis and S1P production of HUVEC cells. After HUVEC cells were transfected with siR-NC (small interfering RNA negative control of SPTLC2), siR-SPTLC2 (small interfering RNA of SPTLC2), SPTLC2-NC (plasmid negative control of SPTLC2) and SPTLC2 (SPTLC2 plasmid), the expression of SPTLC2 protein was detected by western blot assay (A) and their proliferation (B), migration (C), adhesion (D), angiogenesis (E) and S1P production (F) were measured respectively. $* \mathrm{P}<0.05$, ** $\mathrm{P}<0.01$ versus blank group.

Fig. 4 TP inhibited proliferation, migration, invasion, SPTLC 2 mRNA and protein expression in HepG2 cells. (A) CCK-8 assay was used to determine HepG2 cells proliferation after treated with TP $(0,1,2,4 \mu \mathrm{M})$ and DMSO (negative control) for 24 and $48 \mathrm{~h}$. After treating HepG2 cells with TP $(0,1,2,4 \mu \mathrm{M})$ and DMSO (negative control) for $24 \mathrm{~h}$, the migration (B), invasion (C), SPTLC2 mRNA (D) and protein (E) expression of HepG2 were measured respectively. $* \mathrm{P}<0.05$, ** $\mathrm{P}<0.01$ versus control group.

Fig. 5 SPTLC2 can affect the proliferation, migration, invasion and S1P production of HepG2 cells. After HepG2 cells were transfected with siR-NC (small interfering RNA negative control of SPTLC2), siR-SPTLC2 (small interfering RNA of SPTLC2), SPTLC2-NC (plasmid negative control of SPTLC2) and SPTLC2 (SPTLC2 plasmid), the expression of SPTLC2 protein was detected by western blot assay (A) and their proliferation (B), migration (C), invasion (D) and S1P production (E) were measured respectively. $* \mathrm{P}<0.05$, ** $\mathrm{P}<0.01$ versus blank group.

Fig. 6 HUVEC cells may induce proliferation, migration and invasion of HepG 2 cells via the S1P-S1PR pathway. (A) The co-culture time of HUVEC and HepG2 were set to 1 to 4 days, CCK-8 assay was used to determine HepG2 cells proliferation. HUVEC and HepG2 cells were co-cultured for $24 \mathrm{~h}$, their migration (B) and invasion (C) were measured respectively. HUVEC and HepG2 cells were co-cultured for 1 to 4 days, the content of S1P (D) in the co-culture system and the expression of S1PR1, S1PR2 and S1PR3 protein (E) in HepG2 cells were measured respectively. $* \mathrm{P}<0.05, * * \mathrm{P}<0.01$ versus non-co-culture or control group.

Fig. 7 TP inhibited the proliferation, migration and invasion of HepG2 cells induced by HUVEC cells. The HUVEC cells were treated with TP $(0,12.5,25,50 \mathrm{nM})$ for $24 \mathrm{~h}$, the medium was changed to remove the drugs influence, and the treated HUVEC cells were co-cultured with HepG2 cells through transwell co-culture chamber. The data showed that when HUVEC cells 
1 were treated with TP, its promoting effects on proliferation (A), migration (B) and invasion (C) of

2 HepG2 cells were significantly inhibited. $* \mathrm{P}<0.05$, ** $\mathrm{P}<0.01$ versus control group. 


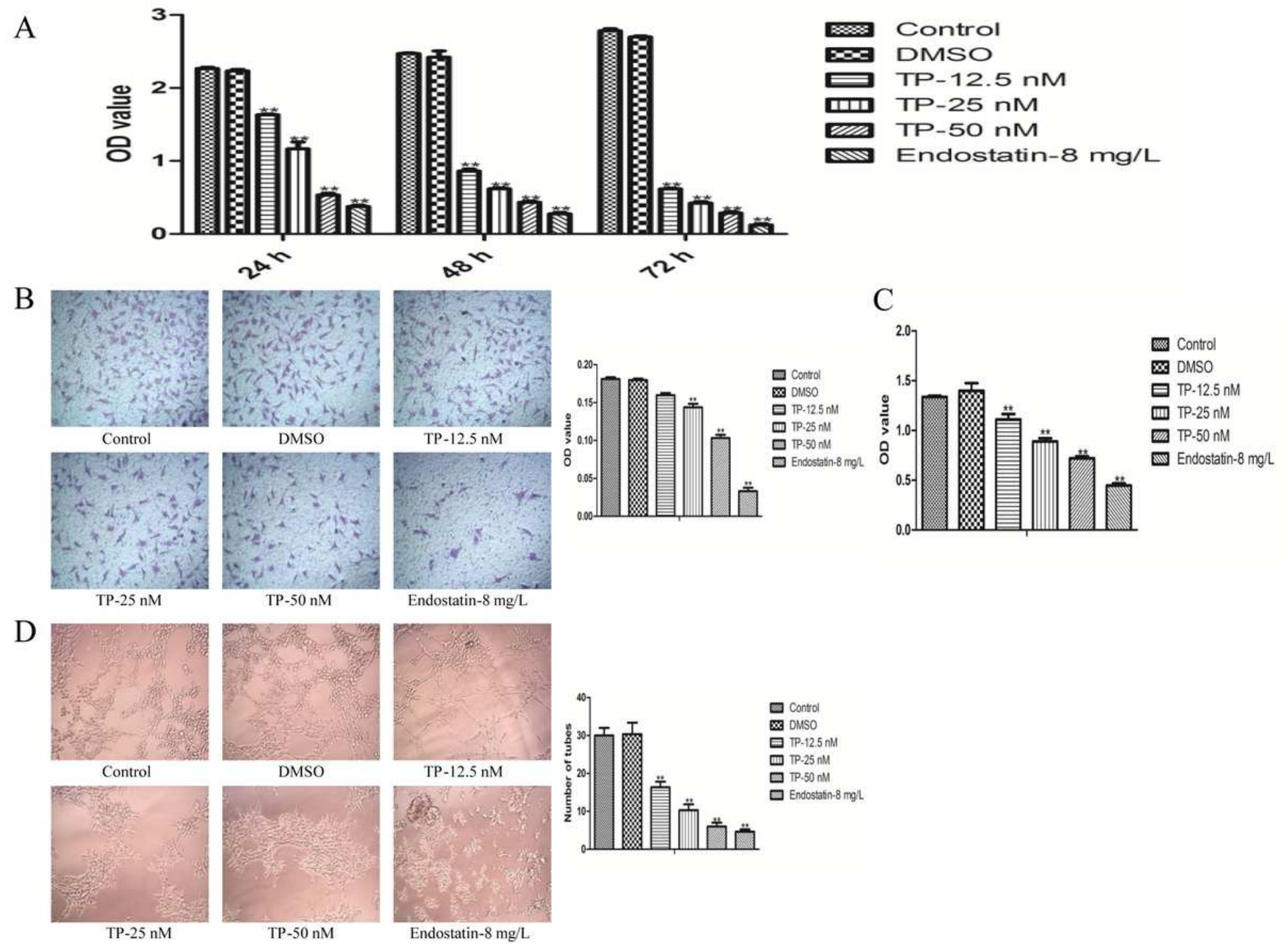

Figure 1

TP inhibited the proliferation, migration, adhesion and angiogenesis of HUVEC cells. (A) CCK-8 assay was used to determine HUVEC cells proliferation after treated with $\operatorname{TP}(0,12.5,25,50 \mathrm{nM})$, DMSO and endostatin (8 mg/L) for 24, 48 and $72 \mathrm{~h}$. After treating HUVEC cells with TP $(0,12.5,25,50 \mathrm{nM}), \mathrm{DMSO}$ and endostatin ( $8 \mathrm{mg} / \mathrm{L})$ for $24 \mathrm{~h}$, the migration (B), adhesion (C) and angiogenesis (D) of HUVEC were measured respectively. * $P<0.05$, ** $P<0.01$ versus control group. 


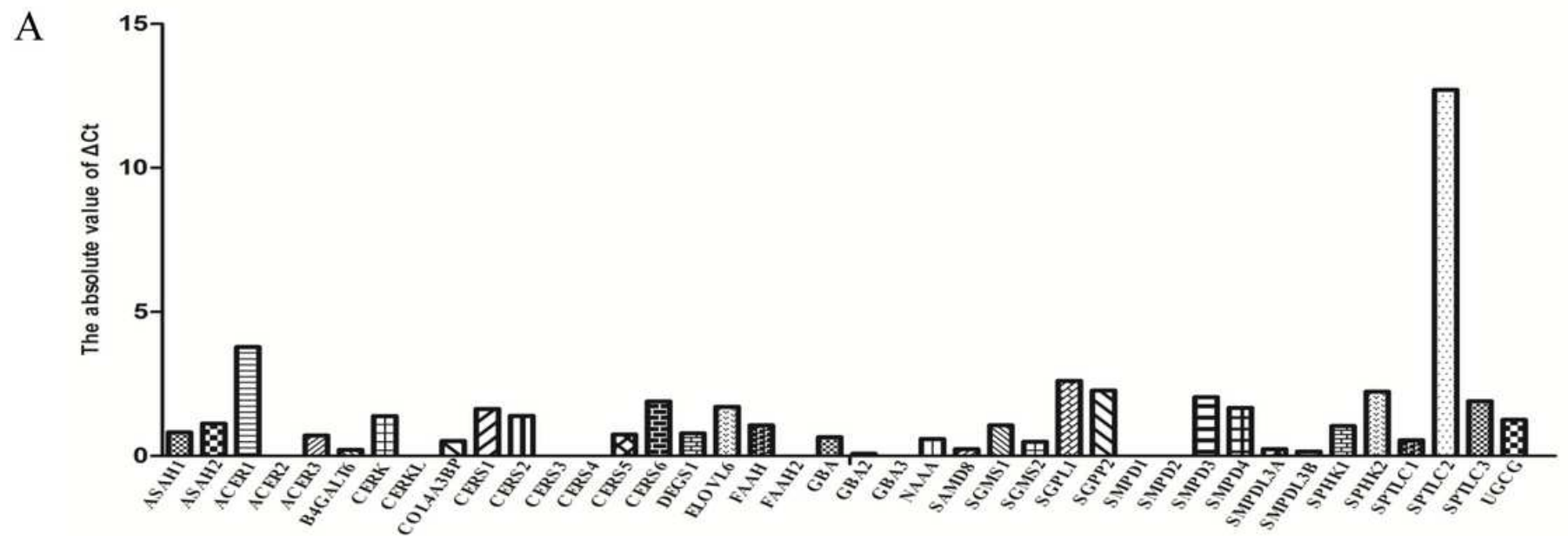

B

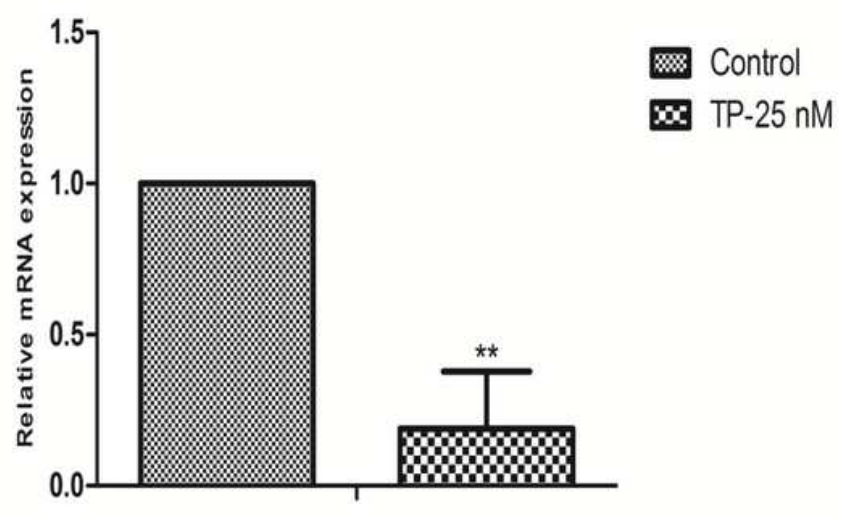

SPTLC2
$\mathrm{C}$
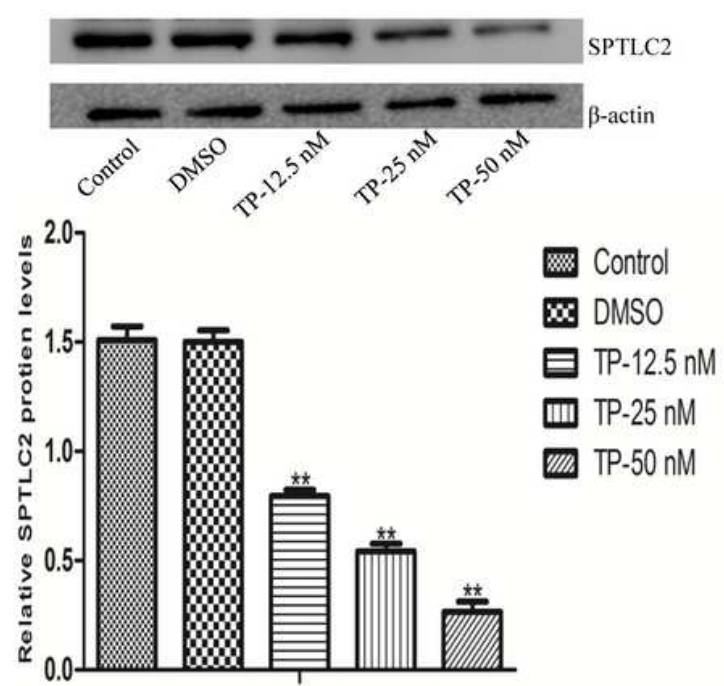

Figure 2

TP significantly down-regulated the expression of SPTLC2 in HUVEC cells. (A) HUVEC cells were treated with TP ( $25 \mathrm{nM})$ for $24 \mathrm{~h}$, and the changes of related genes were screened by PCR Array assay, the data results were the absolute value of $\triangle \mathrm{Ct}$. (B) HUVEC cells were treated with TP (25 nM) for $24 \mathrm{~h}$, and the expression of SPTLC2 mRNA was detected by RT-PCR assay. (C) HUVEC cells were treated with TP $(0$, $12.5,25,50 \mathrm{nM}$ ) and DMSO (negative control) for $24 \mathrm{~h}$, and the expression of SPTLC2 protein in HUVEC cells was detected by western blot assay. ${ }^{*} P<0.05,{ }^{*} P<0.01$ versus control group. 
B

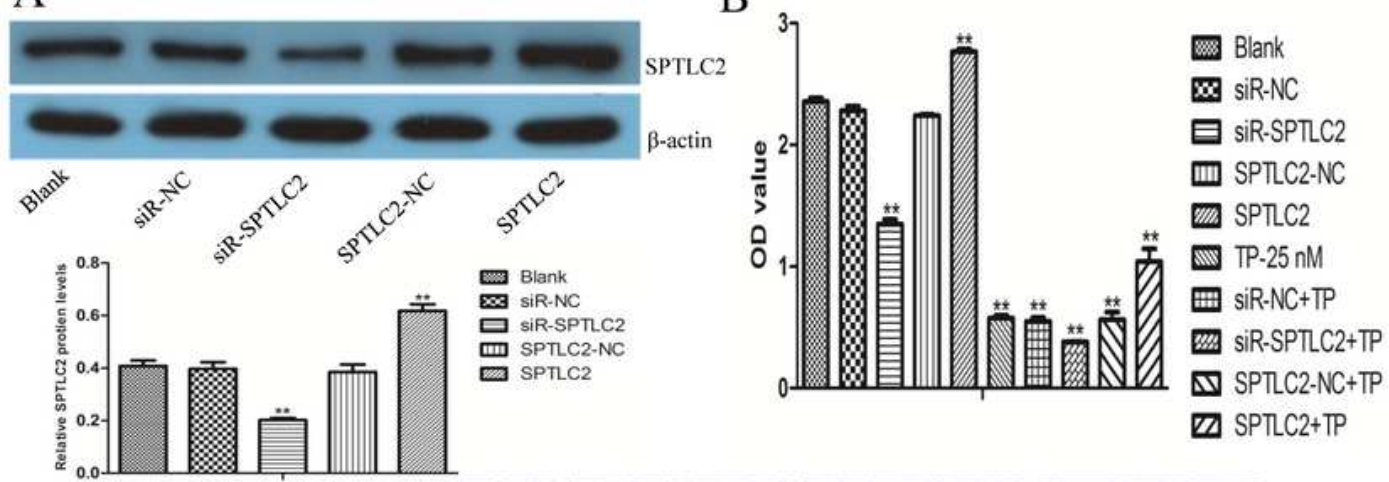

$\mathrm{C}$
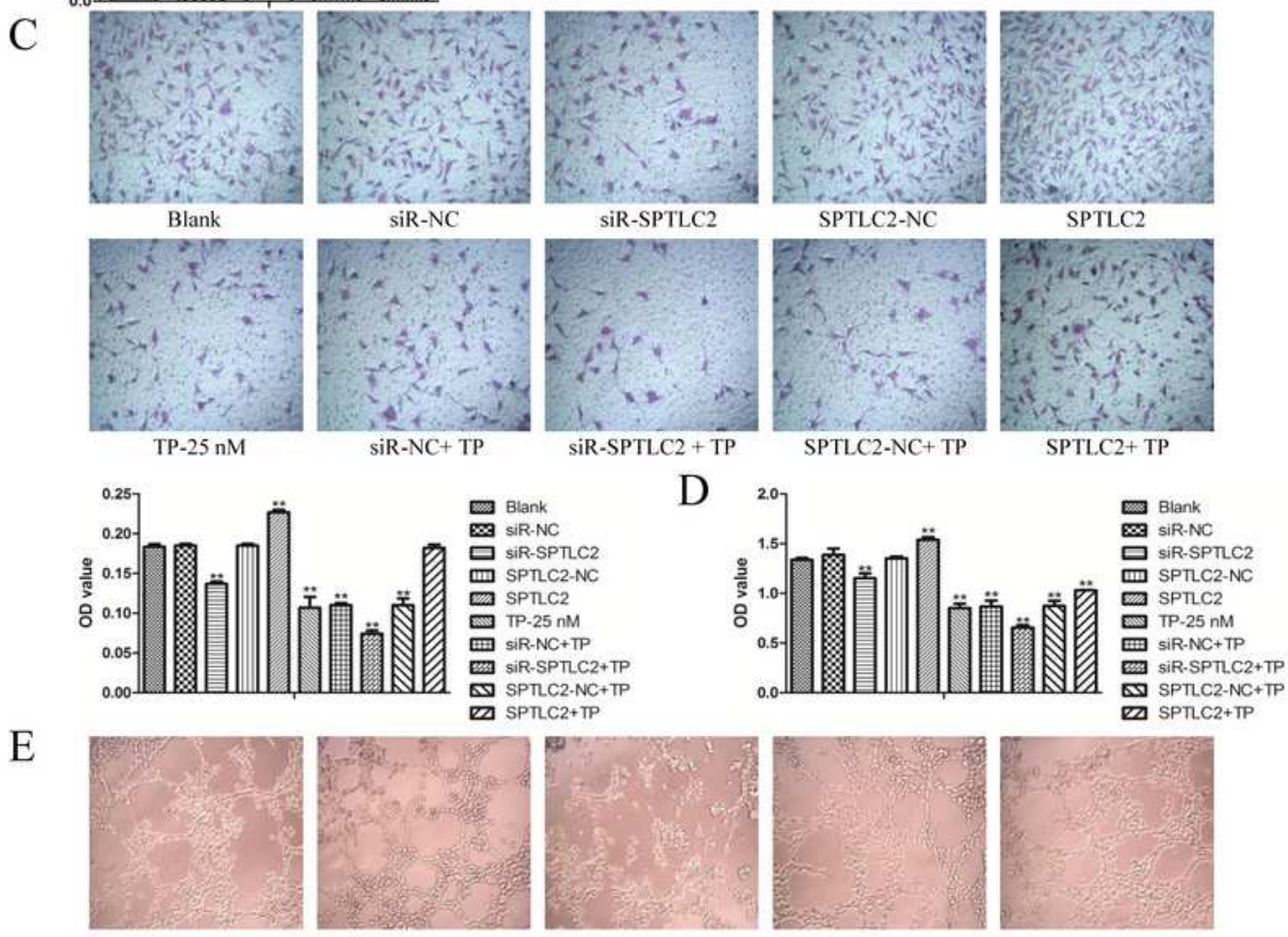

Blank

siR-NC

siR-SPTLC2

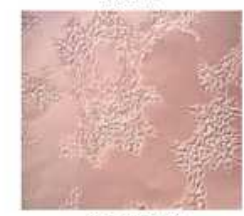

TP- $25 \mathrm{nM}$

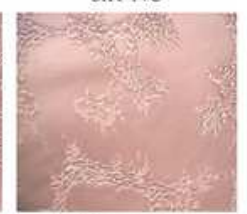

siR-NC+ TP

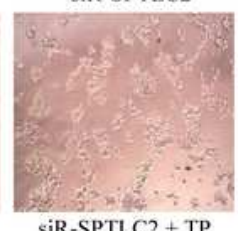

SPTLC2-NC

SPTLC2
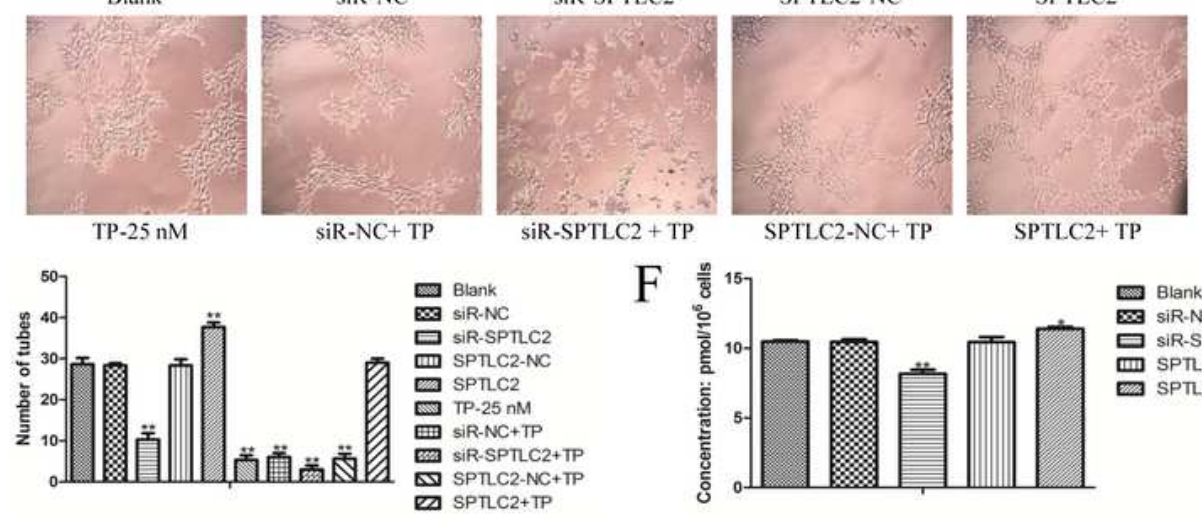

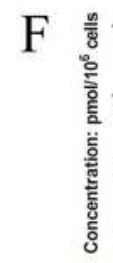

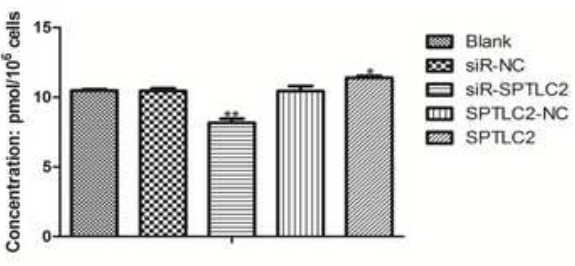

\section{Figure 3}

SPTLC2 can affect the proliferation, migration, adhesion, angiogenesis and S1P production of HUVEC cells. After HUVEC cells were transfected with siR-NC (small interfering RNA negative control of SPTLC2), siR-SPTLC2 (small interfering RNA of SPTLC2), SPTLC2-NC (plasmid negative control of SPTLC2) and SPTLC2 (SPTLC2 plasmid), the expression of SPTLC2 protein was detected by western blot assay (A) and 
their proliferation (B), migration (C), adhesion (D), angiogenesis $(E)$ and S1P production $(F)$ were measured respectively. ${ }^{*} \mathrm{P}<0.05$, ** $\mathrm{P}<0.01$ versus blank group.

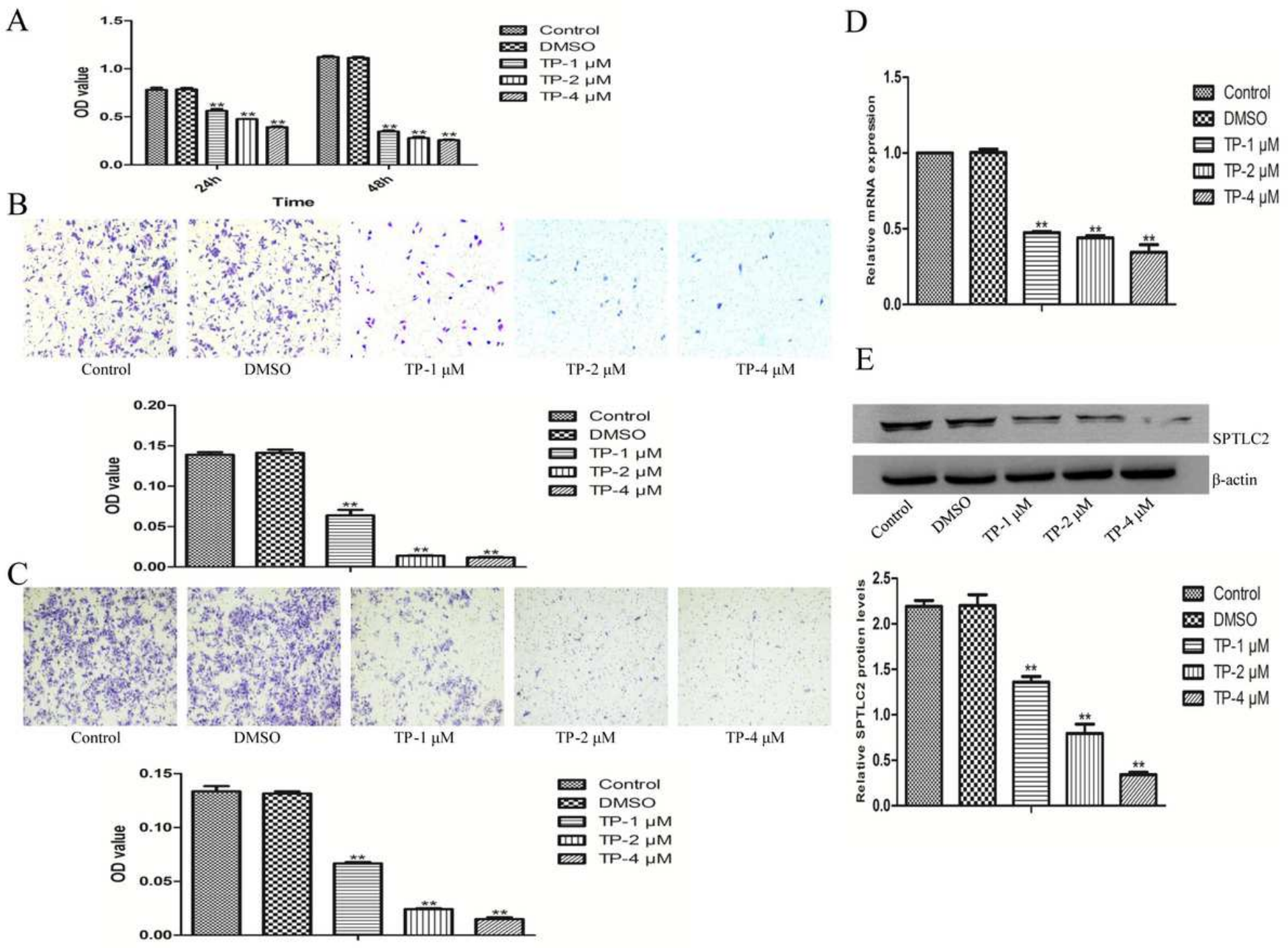

\section{Figure 4}

TP inhibited proliferation, migration囚invasion, SPTLC2 mRNA and protein expression in HepG2 cells. (A) CCK-8 assay was used to determine HepG2 cells proliferation after treated with TP $(0,1,2,4 \mu \mathrm{M})$ and DMSO (negative control) for 24 and $48 \mathrm{~h}$. After treating HepG2 cells with TP $(0,1,2,4 \mu \mathrm{M})$ and DMSO (negative control) for $24 \mathrm{~h}$, the migration (B), invasion (C), SPTLC2 mRNA (D) and protein (E) expression of HepG2 were measured respectively. ${ }^{*} P<0.05$, ${ }^{\star *} P<0.01$ versus control group. 

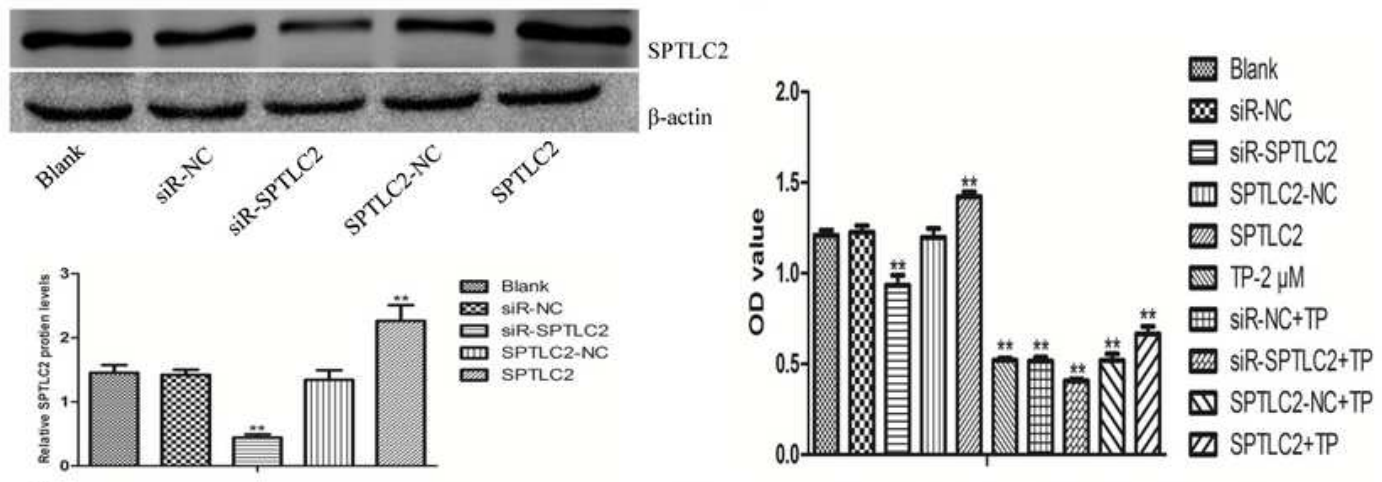

C
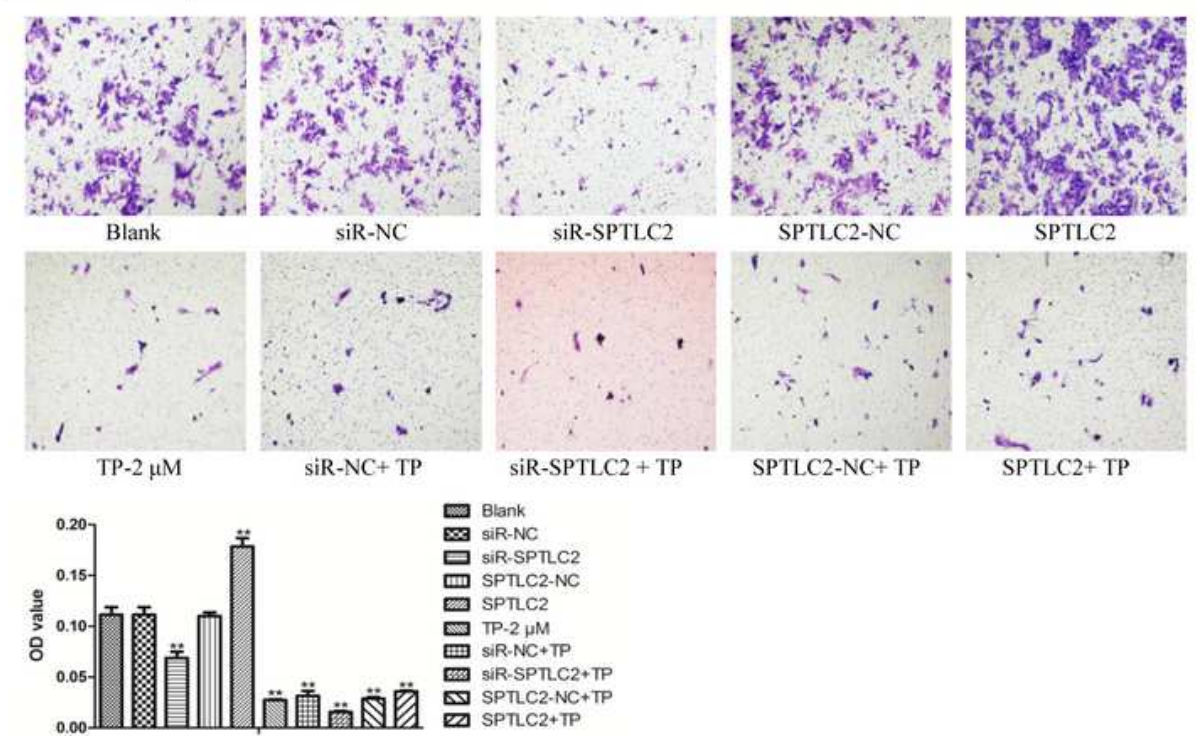

D
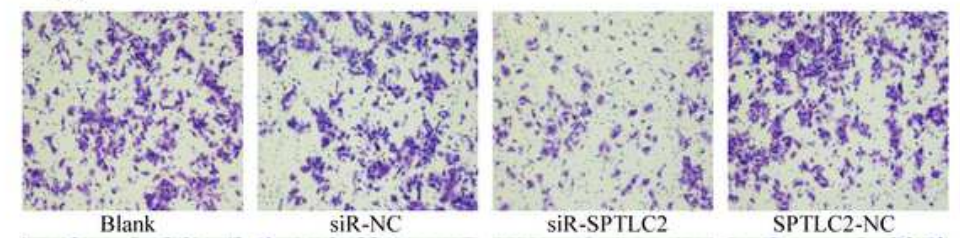

SIR-SPTLC2

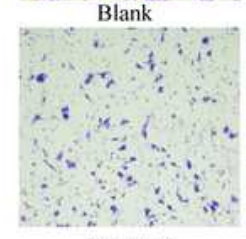

SiR-NC
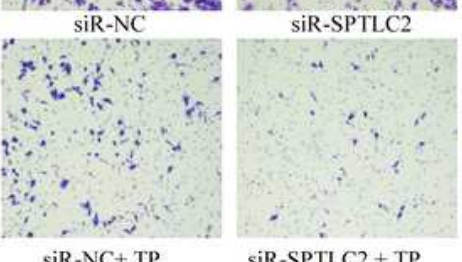

SPTLC2
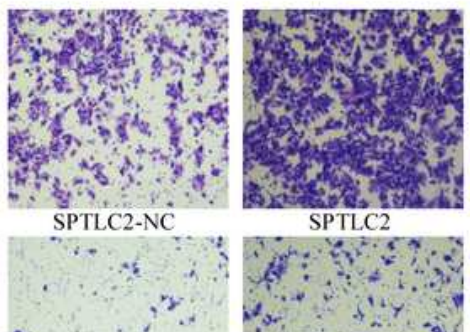

TP-2 uM

siR-NC+ TP
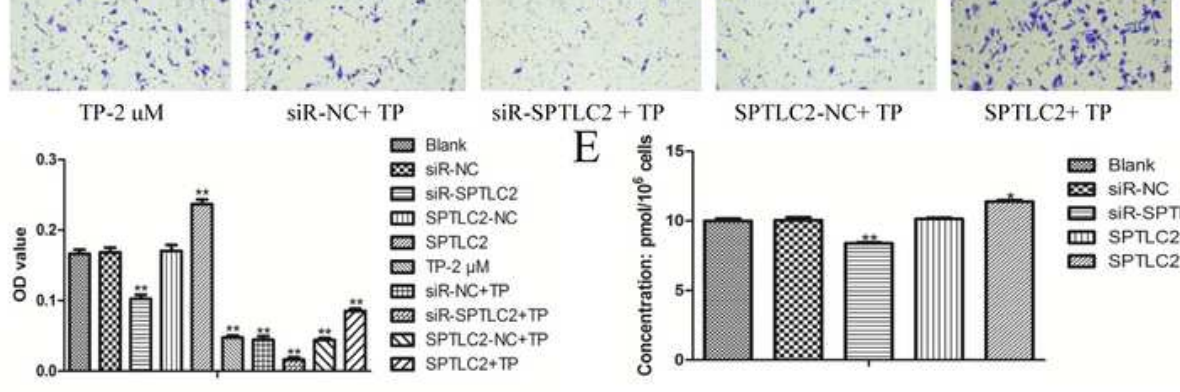

Blank

$\infty$ siR-NC

SiR-SPTLC2

SPTLC2

\section{Figure 5}

SPTLC2 can affect the proliferation, migration, invasion and S1P production of HepG2 cells. After HepG2 cells were transfected with siR-NC (small interfering RNA negative control of SPTLC2), siR-SPTLC2 (small interfering RNA of SPTLC2), SPTLC2-NC (plasmid negative control of SPTLC2) and SPTLC2 (SPTLC2 plasmid), the expression of SPTLC2 protein was detected by western blot assay (A) and their proliferation 
(B), migration (C), invasion (D) and S1P production (E) were measured respectively. * $P<0.05, * \star P<0.01$ versus blank group.

A
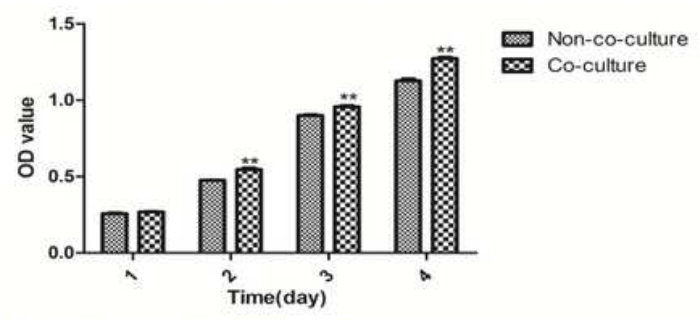

B

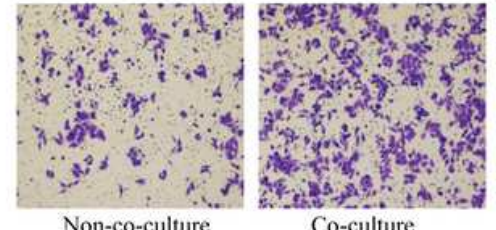

C
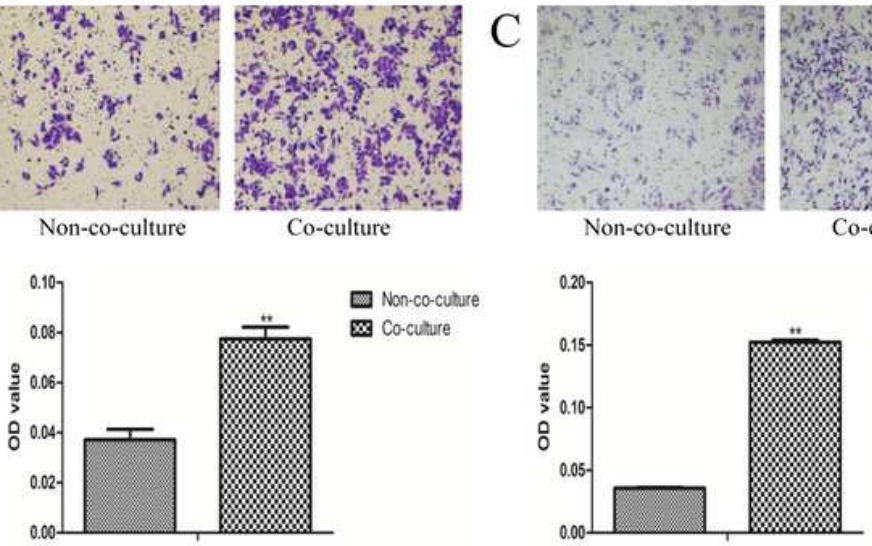

E
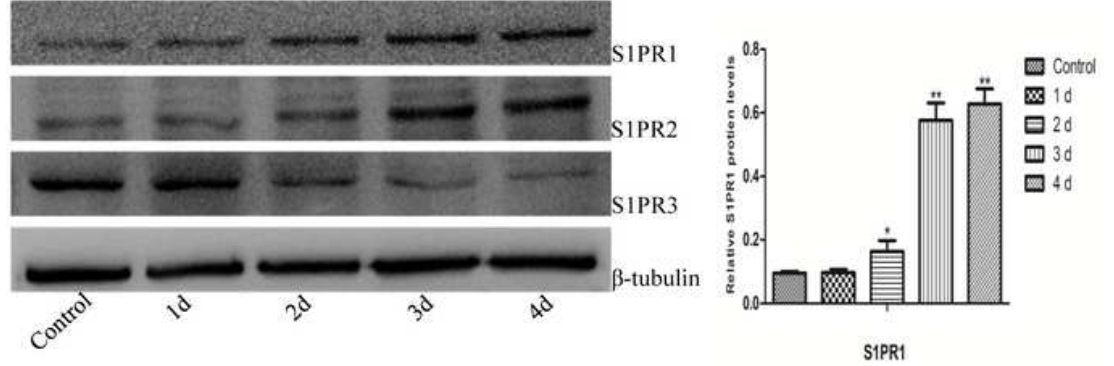

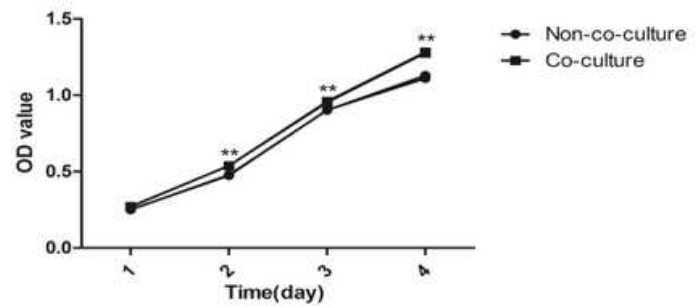

D

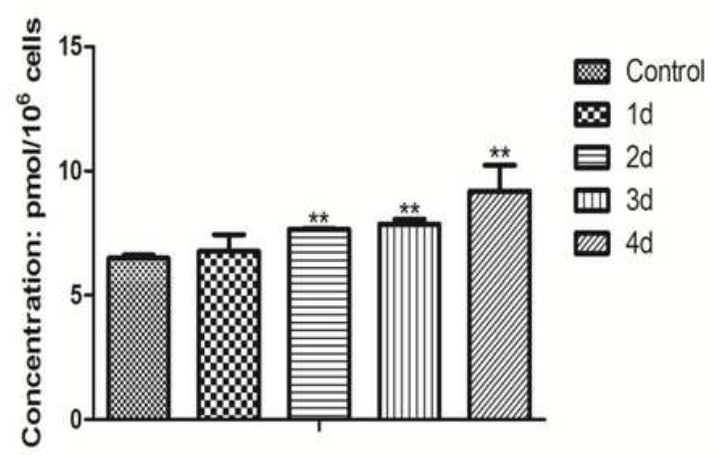

Time(day)

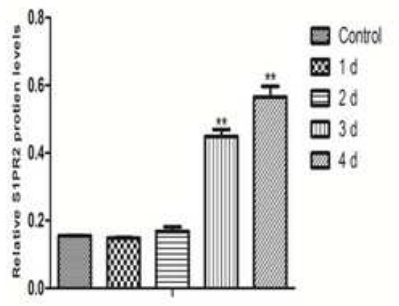

S1PR2

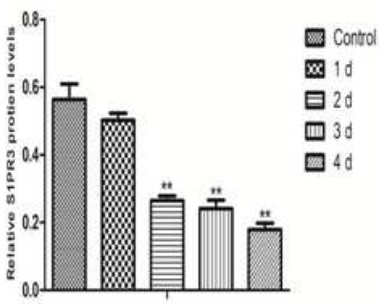

S1PR3

Figure 6

HUVEC cells may induce proliferation, migration and invasion of HepG 2 cells via the S1P-S1PRS pathway. (A) The co-culture time of HUVEC and HepG2 were set to 1 to 4 days, CCK-8 assay was used to determine HepG2 cells proliferation. HUVEC and HepG2 cells were co-cultured for $24 \mathrm{~h}$, their migration (B) and invasion (C) were measured respectively. HUVEC and HepG2 cells were co-cultured for 1 to 4 days, the content of S1P (D) in the co-culture system and the expression of S1PR1, S1PR2 and S1PR3 protein (E) in HepG2 cells were measured respectively. * $P<0.05$, ** $P<0.01$ versus non-co-culture or control group. 
A

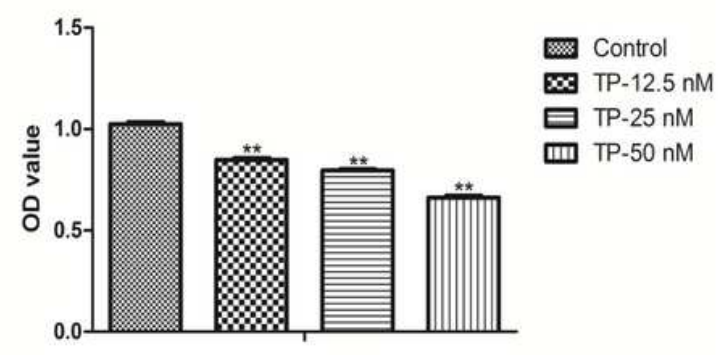

B

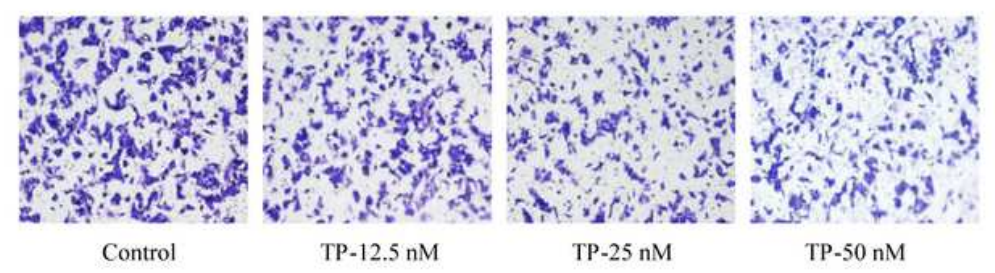

$\mathrm{C}$

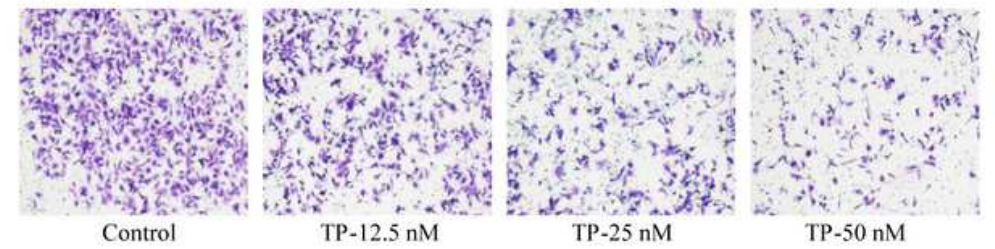

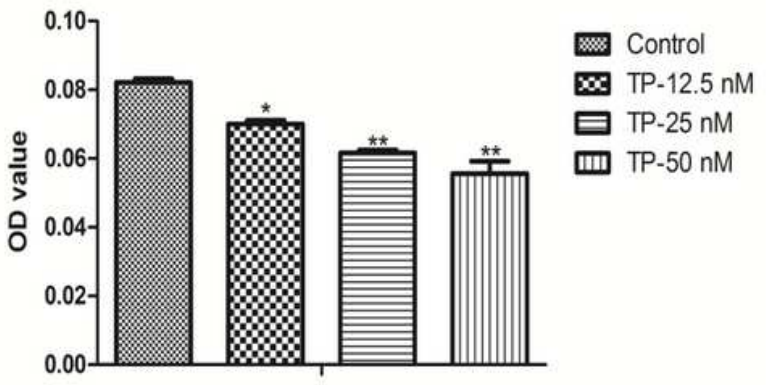

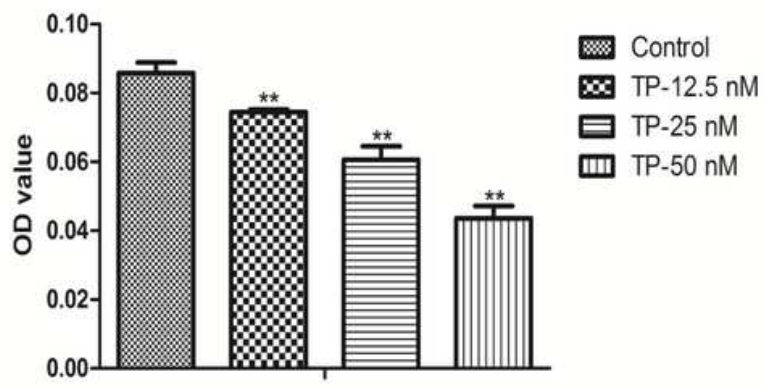

\section{Figure 7}

TP inhibited the proliferation, migration and invasion of HepG2 cells induced by HUVEC cells. The HUVEC cells were treated with $\operatorname{TP}(0,12.5,25,50 \mathrm{nM})$ for $24 \mathrm{~h}$, the medium was changed to remove the drugs influence, and the treated HUVEC cells were co-cultured with HepG2 cells through transwell co-culture chamber. The data showed that when HUVEC cells were treated with TP, its promoting effects on proliferation (A), migration (B) and invasion (C) of HepG2 cells were significantly inhibited. ${ }^{*} P<0.05$, ** $P<0.01$ versus control group. 